\title{
Desenvolvimento e reprodutibilidade de questionário para avaliar práticas e conhecimentos em segurança alimentar de nutricionistas da área clínica
}

\section{Development and reliability of a questionnaire \\ to assess clinical dietitians' practices and \\ knowledge of food safety}

Luísa Helena Maia LEITE ${ }^{1,2}$

William WAISSMANN2,3

Alessandra Bento VEGGI ${ }^{3}$

\section{R E S U M O}

\section{Objetivo}

Os propósitos deste estudo foram desenvolver um questionário para avaliar práticas e conhecimentos em segurança sanitária alimentar, de nutricionistas da área clínica, e medir o nível de reprodutibilidade deste questionário.

\section{Métodos}

O questionário foi desenvolvido a partir de seis áreas temáticas: prevenção da contaminação cruzada; higiene pessoal/ambiental; controle de temperaturas e de alimentos de alto risco e segurança alimentar para indivíduos portadores do vírus da imunodeficiência humana/síndrome da imunodeficiência adquirida. Para medir o nível de reprodutibilidade, utilizou-se o procedimento de teste e re-teste e a estatística kappa simples.

\section{Resultados}

Os resultados mostraram os níveis de reprodutibilidade: $>0,61$ para $95,0 \%$; entre $0,60-0,40$ para $2,5 \%$ e $<0,40$ para $2,5 \%$ das questões testadas. Oitenta por cento das questões apresentaram grau de acertos na faixa de $20,0 \%-80,0 \%$.

\section{Conclusão}

Os resultados mostraram que o instrumento apresenta grau de dificuldade adequado e um nível de reprodutibilidade satisfatório para a maioria das questões (Kappa>0,61), sugerindo que o mesmo representa

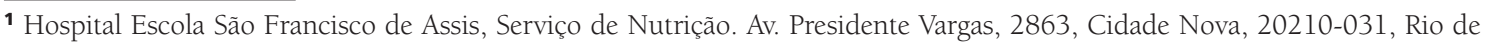
Janeiro, RJ, Brasil. Correspondência para/Correspondence to: L.H.M. LEITE. E-mail: <luisamaia@uol.com.br>.

2 Fundação Oswaldo Cruz, Instituto Nacional de Controle de Qualidade em Saúde. Rio de Janeiro, RJ, Brasil.

${ }^{3}$ Fundação Oswaldo Cruz, Escola Nacional de Saúde Pública Sérgio Arouca. Rio de Janeiro, RJ, Brasil.
} 
uma boa opção para avaliar as práticas e os conhecimentos em segurança alimentar de nutricionistas da área clínica, visando ao planejamento de estratégias educativas.

Termos de indexação: segurança alimentar; síndrome de imunodeficiência adquirida; nutrição; reprodutibilidade de questionário.

\section{A B S T R A C T}

\section{Objective}

The purposes of this study were to develop a questionnaire to assess clinical dietitians' practices and knowledge of food safety and determine the reliability of this instrument.

\section{Methods}

The questionnaire was developed around six areas: prevention of cross contamination, personal and environmental hygiene; control of temperature and high-risk foods and food safety for individuals with human immunodeficiency virus/acquired immunodeficiency syndrome. The reliability of the questionnaire was determined by the test-retest method and simple Kappa statistics.

\section{Results}

The results showed the reliability levels: $>0.61$ for $95.0 \%$, from 0.60 to 0.40 for $2.5 \%$ and $<0.40$ for $2.5 \%$ of the tested questions. Eighty percent of the questions were from $20.0 \%$ to $80.0 \%$ correct.

\section{Conclusion}

The results showed that the instrument presents an adequate difficulty level and a satisfactory reliability level for most of the questions (Kappa>0.61), suggesting that it is a good device to assess clinical dietitians' practices and knowledge of food safety, which allows for the planning of educational strategies.

Indexing terms: food safety; acquired immunodeficiency syndrome; nutrition; reproducibility of questionnaire.

\section{N T R O D U ÇÃ O}

As doenças transmitidas por alimentos (DTA) são, ainda, um importante problema de saúde pública no mundo contemporâneo ${ }^{1-3}$.

Redmond \& Griffith ${ }^{4}$ apontam que muitos casos de DTA poderiam ser evitados, se comportamentos preventivos fossem adotados em toda a cadeia produtiva de alimentos. No entanto, diante da impossibilidade de produzir alimentos totalmente isentos de patógenos, os cuidados na manipulação dos alimentos, nos domicílios, representam, hoje, importante etapa para reduzir a incidência dessas doenças ${ }^{5,6}$. Entre as medidas preventivas, a educação sanitária da população é apontada como uma estratégia para redução dos casos de DTA, complementando as atividades governamentais de inspeção e regulação ${ }^{7-9}$. As informações preventivas são particularmente importantes para os grupos vulneráveis às DTA ou populações a risco. Entre estes, destacam-se os idosos, as gestantes, os imunodeprimidos, como os portadores do vírus da imunodeficiência humana (HIV) ou da Síndrome da imunodeficiência adquirida (AIDS), e usuários de drogas imunossupressoras (quimioterapia) ${ }^{10}$.

Mensagens educativas sobre higiene e segurança alimentar poderiam ser transmitidas em unidades de saúde ambulatoriais como parte das ações de prevenção e promoção da saúde ${ }^{11}$. O conteúdo dessas mensagens poderia ter como alvo a prevenção de enteropatógenos de alta gravidade para imunodeprimidos e a prevenção de falhas de manipulação de alimentos mais comuns entre os consumidores ${ }^{12,13}$.

Segundo Woteki et al. ${ }^{14}$, Nutrição e Segurança Alimentar estão intimamente ligadas, devendo a educação sanitária fazer parte das atividades de aconselhamento nutricional e da dietoterapia.

O cenário descrito indica a necessidade de avaliar se os profissionais de saúde, em especial 
se nutricionistas de unidades ambulatoriais têm adotado, na prática clínica, intervenções educativas que possam prevenir os riscos de DTA em sua clientela, particularmente as populações vulneráveis, bem como avaliar o grau de conhecimentos dos profissionais sobre o tema.

A literatura científica disponível indica que há escassez de instrumentos validados sobre segurança alimentar, que tenham sido desenvolvidos para nutricionistas da área clínica ${ }^{15}$. Os propósitos deste estudo foram desenvolver um questionário para medir as práticas e os conhecimentos em segurança sanitária alimentar, de nutricionistas da área clínica e medir o nível de reprodutibilidade deste instrumento.

\section{MÉ TO D OS}

Para o desenho do estudo foi selecionada uma amostra de conveniência, composta por 20 nutricionistas da área clínica, de 2 hospitais públicos, do município do Rio de Janeiro. Foram excluídos os profissionais nutricionistas da área de produção de refeições dos hospitais em estudo. A amostra foi estimada, levando-se em consideração o total de nutricionistas a serem entrevistados ( $n=27)$, em uma amostra de 14 unidades ambulatoriais, de tratamento de HIV/AIDS, selecionadas para o estudo final.

Para a realização do estudo, foi obtida a autorização formal dos diretores e coordenadores das unidades hospitalares, após a avaliação ética pelo Comitê de Ética e Pesquisa da Secretaria Municipal de Saúde do Rio de Janeiro, segundo a Resolução 196/96. O projeto de pesquisa recebeu o parecer aprovado em 24 de novembro de 2003.

Para construir o questionário, foram adotadas seis dimensões em segurança alimentar: prevenção da contaminação cruzada; higiene pessoal/higiene ambiental; controle de temperaturas e controle de alimentos de alto risco, segundo Medeiros et al. ${ }^{12}$, e questões sobre segurança alimentar de importância especial para portadores de HIV/AIDS ${ }^{13,16}$.
Inicialmente, o instrumento foi submetido à avaliação crítica de 5 nutricionistas com atuação na área de segurança alimentar (3 professores universitários; 1 profissional de inspeção e 1 consultor em segurança alimentar).

Em seguida, o instrumento preliminar foi submetido a uma pré-testagem, visando a avaliar a clareza, o grau de compreensão, a pertinência das questões e o nível de concordância das respostas. Participaram dessa etapa preliminar 20 nutricionistas, com atuação na área clínica, de um hospital universitário. Os questionários foram aplicados no local de trabalho e re-aplicados num intervalo de 30 dias. Após essas etapas, o instrumento foi reformulado com base nas sugestões dos revisores e nos resultados do pré-teste.

A versão final do instrumento continha 37 questões, divididas em 6 blocos: informações pessoais; prevenção de DTA no ambiente clínico; conhecimentos sobre contaminação cruzada; higiene pessoal/ambiental; controle de temperaturas e de alimentos de alto risco e segurança alimentar e HIV/AIDS. Em relação às categorias de respostas, questões sobre conhecimentos foram divididas em dicotômicas (sim/não) ou de múltipla escolha; questões sobre percepção de risco variaram de "alto" a "nenhum risco"; questões sobre práticas de segurança alimentar variaram de "sempre" a "nunca adota as práticas recomendadas".

A versão final do questionário foi aplicada no local de trabalho, sob a forma de auto-preenchimento, no período de julho a agosto de 2004, supervisionado por um único pesquisador, utilizando-se o procedimento teste e re-teste, com intervalo de 15 dias entre a primeira e a segunda aplicação.

Para estimar o nível de concordância optouse por utilizar a medida do coeficiente Kappa simples, pois o formato das respostas não permitiu o cálculo do kappa ponderado. Utilizaram-se como referência para os valores de concordância as orientações da literatura especializada ${ }^{17}$ : coeficiente Kappa <0,10 (ausência de concordância); de 0,11 a 0,40 (fraca); 0,41-0,60 (discreta); de 
0,61-0,80 (moderada); de 0,81-0,99 (substancial) e 1,0 (concordância perfeita). Cabe destacar que, em geral, são recomendadas maior ênfase ao valor numérico e certa cautela ao interpretar os resultados, pois os valores de referência das escalas podem variar entre os autores ${ }^{18}$. Dessa forma, neste estudo, foram seguidas as recomendações de Ravid ${ }^{19}$, que destaca que em estudos exploratórios é desejável um nível de concordância maior que 0,61 , entretanto níveis entre $0,50-0,60$ são também considerados aceitáveis, devendo-se eliminar somente as questões com Kappa $<0,20$ ou aquelas com efeito negativo.

As respostas corretas foram definidas considerando-se a legislação sanitária vigente sobre Boas Práticas de Fabricação de Alimentos, sendo selecionadas duas portarias (CVS-6/99 do Estado de São Paulo; Portaria 2535/03 do Município de São Paulo) ${ }^{20,21}$ e recomendações de importância especial para portadores de HIV/AIDS ${ }^{13,16}$.

O grau de dificuldade do instrumento foi estimado segundo Medeiros et al. ${ }^{22}$, utilizando-se como referência a faixa de $20-80 \%$ de acertos.

Para a análise dos resultados, foi criado um banco de dados utilizando-se o programa Epi Info for Windows, em seguida foi analisada a estatística kappa simples, utilizando-se o programa STATA versão 7.0.

\section{RES ULTADOS}

Participou do estudo de reprodutibilidade do instrumento final, um grupo de 20 nutricionistas com atuação na área clínica, constituído, em sua maioria (95\%), por mulheres, na faixa etária de 29 a 35 anos (60\%). Cinqüenta por cento possuía até 10 anos de formação profissional e 70\% trabalhava há menos de 5 anos com portadores de HIVIAIDS.

De forma geral, os resultados obtidos no estudo de reprodutibilidade do questionário mostraram, para 95\% das questões avaliadas, um nível de concordância considerado satisfatório $(>0,61)$, moderado para $2,5 \%(0,60-0,40)$ e discreto para $2,5 \%(<0,40)$. Nenhuma questão apresentou reprodutibilidade inferior a 0,20 ou com efeito negativo (Tabela 1).

Duas questões, situadas no bloco de questões sobre segurança alimentar e HIV/AIDS, apresentaram nível de reprodutibilidade abaixo de 0,60 . Em uma delas o nível de concordância medido foi $k=0,53$, sendo essa questão relacionada à prática de orientação educativa para o reaquecimento de alimentos prontos para o consumo (delivery). Para a outra questão (relacionada com o conhecimento sobre práticas de segurança alimentar para prevenir criptosporidiose), a concordância medida foi $k=0,35$.

Quatro questões encontravam-se na faixa de acertos inferior a $20 \%$, sendo avaliadas de alto grau de dificuldade e relacionadas, respectivamente, com o correto procedimento para higienizar vegetais crus ( $5 \%$ de acertos); orientações sobre a presença de animais no ambiente doméstico $(17 \%)$; conservação de ovos na geladeira doméstica (15\%) e prevenção de criptosporidiose (nenhum acerto).

\section{I S C U S S Ã O}

O estudo de desenvolvimento de um questionário sobre segurança alimentar e HIV/AIDS, para nutricionistas da área clínica, foi uma iniciativa para suprir as necessidades da literatura científica nacional sobre a existência de um instrumento culturalmente adequado, que tenha tido sua reprodutibilidade testada, favorecendo a coleta de dados científicos válidos e confiáveis.

A determinação dos níveis de reprodutibilidade de um questionário representa um aspecto de grande importância, tendo em vista que esses níveis estão estritamente associados à qualidade dos dados que serão coletados ${ }^{18,23}$.

O questionário avaliado apresentou um nível de concordância satisfatório para a maioria das questões (95\%), o que indica alto nível de estabilidade do instrumento. Resultados similares foram encontrados por outros autores, como 
Tabela 1. Seções e níveis de reprodutibilidade de questionário para medir práticas e conhecimentos em segurança sanitária alimentar de nutricionistas de hospitais públicos da cidade do Rio de Janeiro, março a julho de 2004. (n=20).

\begin{tabular}{|c|c|c|}
\hline Seções do questionário & Valores Kappa & IC (95\%) \\
\hline \multicolumn{3}{|l|}{ Prevenção de DTA no ambiente clínico } \\
\hline $\begin{array}{l}\text { Faz parte do tratamento dietoterápico fornecer informações educativas, nas consultas ambulatoriais } \\
\text { sobre a prevenção de DTA? }\end{array}$ & 1,0 & - \\
\hline $\begin{array}{l}\text { Com que freqüência você transmite, nas consultas ambulatoriais, informações para prevenção de } \\
\text { DTA? }\end{array}$ & 0,93 & $0,80-1,05$ \\
\hline $\begin{array}{l}\text { Qual a porcentagem aproximada de seus clientes ambulatoriais composta por idosos, gestantes, } \\
\text { crianças e portadores de HIVIAIDS? }\end{array}$ & 0,68 & $0,43-0,93$ \\
\hline $\begin{array}{l}\text { Se você tivesse de dar uma nota para os conhecimentos dos seus pacientes sobre práticas de higiene } \\
\text { e segurança alimentar, ela seria? }\end{array}$ & 1,0 & - \\
\hline $\begin{array}{l}\text { Se você tiver de dar uma nota para os seus conhecimentos atuais sobre práticas de higiene e segurança } \\
\text { alimentar, ela seria? }\end{array}$ & 0,91 & $0,69-1,13$ \\
\hline \multicolumn{3}{|l|}{ Conhecimentos sobre contaminação cruzada } \\
\hline O termo "contaminação cruzada" está relacionado com...? & 1,0 & - \\
\hline Qual prática preventiva é mais eficiente para minimizar os risco de "contaminação cruzada"? & 1,0 & - \\
\hline $\begin{array}{l}\text { Em relação ao uso de superfícies de corte, qual a prática mais eficiente para minimizar os riscos de } \\
\text { contaminação cruzada? }\end{array}$ & 0,70 & $0,44-0,97$ \\
\hline $\begin{array}{l}\text { Você conhece algum alimento para o qual, atualmente, seja obrigatória, no Brasil, a presença de } \\
\text { informações sobre prevenção da contaminação cruzada em sua rotulagem? }\end{array}$ & 0,64 & $0,16-1,45$ \\
\hline \multicolumn{3}{|l|}{ Conhecimentos sobre higiene pessoal, ambiental e de alimentos } \\
\hline Para higienizar vegetais a serem consumidos crus, o procedimento mais eficiente seria? & 1,0 & - \\
\hline Qual a concentração ideal de hipoclorito por litro de água, na higienização de vegetais crus? & 0,69 & $0,32-1,05$ \\
\hline Qual o momento mais indicado para a lavagem das mãos durante o preparo dos alimentos? & 0,88 & $0,58-1,16$ \\
\hline Qual a recomendação sobre a presença de animais no ambiente doméstico? & 0,78 & 0,53-1,03 \\
\hline \multicolumn{3}{|l|}{ Conhecimentos sobre controle de temperaturas } \\
\hline Qual a faixa de temperatura ideal para conservar alimentos em geladeira doméstica? & 0,92 & $0,78-1,04$ \\
\hline Para carnes e aves cruas, conservadas sob refrigeração, qual o prazo de validade mais indicado? & 0,65 & $0,34-0,95$ \\
\hline Ao descongelar carnes e aves, qual seria o procedimento de descongelamento mais indicado? & 0,62 & $012-1,12$ \\
\hline $\begin{array}{l}\text { Para reaproveitar sobras de alimentos prontos à base de carnes ou aves, qual seria o procedimento de } \\
\text { conservação mais indicado? }\end{array}$ & 0,76 & 050-1,02 \\
\hline $\begin{array}{l}\text { Sobre o armazenamento de ovos no refrigerador doméstico, qual o compartimento mais indicado } \\
\text { para a conservação segura? }\end{array}$ & 0,85 & $0,65-1,05$ \\
\hline Quais os atributos indicam a melhor qualidade sanitária de ovos? & 0,66 & $0,33-0,98$ \\
\hline \multicolumn{3}{|l|}{ Segurança alimentar e HIVIAIDS } \\
\hline $\begin{array}{l}\text { Dos pacientes sob a sua assistência ambulatorial, qual é a porcentagem aproximada de portadores de } \\
\text { HIVIAIDS? }\end{array}$ & 0,88 & $0,56-1,19$ \\
\hline Qual o \% aproximado de portadores de HIVIAIDS que, solicita informações sobre higiene alimentar? & 1,0 & - \\
\hline $\begin{array}{l}\text { Grupo de portadores de HIVIAIDS que você considera prioritário para receber informações para a } \\
\text { prevenção de DTA? }\end{array}$ & 0,85 & $0,60-1,09$ \\
\hline Qual o risco de portadores de HIVIAIDS contraírem DTA em suas residências? & 0,75 & $0,53-0,97$ \\
\hline Qual o risco de portadores de HIVIAIDS contraírem DTA fora de suas residências? & 0,92 & $0,77-1,07$ \\
\hline Qual a mais eficiente medida para prevenir as DTA de alta gravidade, para portadores de HIVIAIDS? & 0,76 & $0,50-1,00$ \\
\hline $\begin{array}{l}\text { Identifique o erro ou falha no preparo dos alimentos relacionados com a transmissão de Noroviroses, } \\
\text { Shigella sp. e Hepatite A? }\end{array}$ & 0,78 & $0,57-0,97$ \\
\hline Qual é a mais eficiente medida para prevenir Criptosporidiose? & 0,35 & $-0,07-1,01$ \\
\hline Com que freqüência você orienta pacientes HIV-positivos para não consumirem ovos crus/mal cozidos? & 0,79 & $0,55-1,01$ \\
\hline Com que freqüência você orienta pacientes HIV-positivos para reaquecerem alimentos delivery? & 0,53 & $0,20-0,85$ \\
\hline
\end{tabular}


Scheule ${ }^{24}$, em um estudo americano, ao desenvolver um questionário para avaliar conhecimentos sobre segurança alimentar de diferentes profissionais de saúde que atuam na área maternoinfantil, verificando um nível de reprodutibilidade superior a 0,60 para a maioria das questões. Em outro inquérito, destinado a estudantes de nutrição e hotelaria ${ }^{25}$, também se encontrou resultado semelhante, assim como no estudo de Strohbehn ${ }^{26}$, ao avaliar os conhecimentos sobre segurança alimentar de nutricionistas da área de produção de refeições coletivas.

Apesar de os inquéritos citados apresentarem reprodutibilidade adequada, tais instrumentos não foram desenvolvidos especificamente para nutricionistas da área clínica que assistem populações vulneráveis às DTA, como os portadores de HIV/AIDS. Além disso, a tradução de instrumentos desenvolvidos em outros países, envolvendo questões sobre alimentação e nutrição, pode apresentar limitações, quando aplicados em populações que apresentam características culturais diferentes ${ }^{15}$.

No instrumento avaliado, duas questões apresentaram reprodutibilidade abaixo de 0,60, estando situadas no bloco de questões sobre segurança alimentar e HIVIAIDS. Acredita-se que a concordância mais baixa tenha sido influenciada pelo fato de os nutricionistas que participaram do estudo, apesar de atuarem na área clínica, não estarem satisfatoriamente familiarizados com questões mais específicas de segurança alimentar e HIVIAIDS, ou mesmo pelo fato de assistirem, ainda, uma parcela muito pequena dessa clientela. Pela relevância das duas questões para este estudo, optou-se pela manutenção das mesmas no questionário.

Recomenda-se como adequado, para os estudos exploratórios, um nível de concordância superior a 0,61, devendo-se eliminar questões com níveis de reprodutibilidade abaixo de 0,20 ou aquelas com efeito negativo ${ }^{19}$. Esses parâmetros foram considerados, por outros autores, em estudos de confiabilidade de inquéritos para medir conhecimentos e comportamentos em segurança alimentar ${ }^{27-29}$, e adotados como referência para análise dos resultados deste estudo.

Um reconhecido fator, que pode interferir nas estimativas de reprodutibilidade em estudos do tipo teste e re-teste, que avaliam práticas e comportamentos alimentares, é o intervalo de tempo decorrido entre a primeira e a segunda entrevista. Se o intervalo for muito longo há grandes chances de mudanças de comportamento; por outro lado, se o intervalo for muito curto, a familiaridade das questões pode gerar resultados não confiáveis ${ }^{30}$.

Neste estudo, os resultados obtidos na etapa da testagem preliminar do instrumento revelaram que o intervalo de 30 dias para o reteste do instrumento pode ter afetado o nível de reprodutibilidade das questões, já que mais de $80 \%$ encontravam-se abaixo de 0,60. Mediante estes resultados, optou-se por reduzir o intervalo de tempo para 15 dias, no estudo de reprodutibilidade da versão final do instrumento. Esse intervalo já havia sido adotado, anteriormente, por outros autores, com resultados satisfatórios 22,28.

Outro fator que pode influenciar a estabilidade de um instrumento é o grau de dificuldade das questões, sendo recomendada uma faixa de acertos de $20-80 \%{ }^{22}$.

Os resultados deste estudo mostraram que a reprodutibilidade de uma questão pode ter sido afetada pelo grau de dificuldade, que se situou abaixo de $20 \%$. A falta de conhecimento sobre práticas preventivas para evitar criptosporidiose pode ser identificada pela alta freqüência de respondentes (75\%) que escolheram a opção de resposta "não sei".

O mesmo efeito não foi observado para outras 3 questões com grau de acertos inferior a $20 \%$, relativas a procedimentos recomendados pela legislação sanitária vigente para higienização de vegetais crus; reaproveitamento de sobras e prevenção de zoonoses em imunodeprimidos ${ }^{16}$.

Os resultados encontrados sugerem que o instrumento testado apresenta um nível de reprodutibilidade satisfatório, sendo uma boa opção 
para o levantamento de informações de conhecimentos, percepções e práticas em segurança sanitária alimentar de nutricionistas da área clínica, na busca de informações que possam servir como base para o planejamento de intervenções educativas destinadas às populações vulneráveis, particularmente, portadores de HIVIAIDS. Este instrumento pode ser útil, também, para avaliar resultados de programas de treinamentos em segurança alimentar para profissionais de saúde. Entretanto, é desejável que sejam realizados estudos futuros que contemplem outros aspectos da confiabilidade e validade visando ao aprimoramento deste instrumento.

\section{COLABORADORES}

L.H.M. LEITE realizou a revisão bibliográfica, construção do instrumento, trabalho de campo, análise e discussão dos resultados. A.B. VEGGI colaborou na análise e discussão dos resultados. W. WAISSMANN coordenou as etapas de construção do instrumento, discussão e análise dos resultados. Todos os autores participaram da elaboração da versão final do artigo.

\section{REFER Ê NCIAS}

1. Kosec M, Bern C, Guerrant RL. The global burden of diarrhoeal disease, as estimated from studies published between 1992-2000. Bull World Health Organ. 2003; 81(3):197-204.

2. Guerrant RL, Kosec M, Moore S, Lorntz B, Brantley $\mathrm{R}$, Lima AAM. Magnitude and impact of diarrheal diseases. Arch Med Res. 2002; 33(4):351-5.

3. Farthing MJG. Diarrhoea: a significant worldwide problem. Int J Antimicrob Agents. 2000; 14(1):65-9.

4. Redmond EC, Griffith CJ. Consumer food handling in the house: a review of food safety studies. J Food Prot. 2003; 66(1):130-61.

5. Scott E. Developing a rational approach to hygiene in the domestic setting. J Infect. 2001; 43(1):45-9.

6. Bloomfield S. Gastrointestinal diseases in the domestic: setting what are the issues? J Infect. 2001; 43(1):23-9.

7. Liang AP, Koopmans M, Doyle MP, Bernard DT, Brewer CE. Teaming up to prevent foodborne disease. Emerg Infect Dis. 2001; 7(3):533-4.
8. McCabe-Sellers BJ, Beattie SE. Food safert emerging trends in foodborne illness surveillance and prevention. J Am Diet Assoc. 2004; 104(11):1708-17.

9. Woteki CE, Kineman B. Challenges and approaches to reducing foodborne illness. Ann Rev Nutr. 2003; 23(3):315-44.

10. Gerba CP, Rose JB, Haas CN. Sensitive populations: who is the greatest risk? Int J Food Microbiol. 1996; 30(1-2):113-23.

11. Abdussalam M, Kaferstein FK. Food safety in primary health care. World Health Forum. 1994; 15(4):393-9.

12. Medeiros LC, Hillers VN, Kendall PA, Mason A. Food safety education: what should we be teaching to consumers? J Nutr Educ. 2001; 33(2):108-13.

13. Kendall P, Medeiros LC, Hillers V, Chen G, DiMascola S. Food handling behaviors of special importance for pregnant women, infants and young children, the elderly, and immune-compromised people. J Am Diet Assoc. 2003; 103(12):1646-9.

14. Woteki CE, Facinoli SC, Schor D. Keep food safe to eat: healthful food must be safe as well as nutritious. J Nutr. 2001; 131(Suppl 1):502-9.

15. Medeiros LC, Hillers VN, Kendall PA, Mason A. Evaluation of food safety education for consumers. J Nutr Educ. 2001; 33(1):27-34.

16. Hayes C, Elliot E, Krales E, Downer G. Food and water safety for persons infected with human immunodeficiency virus. Clin Infect Dis. 2003; 36(Suppl 2):106S-9S.

17. Shout PE. Measurement reliability and agreement in psychiatry. Stat Methods Med Res. 1998; 7(3):301-17.

18. Campos MR, Leal MC, Souza Jr. PR, Cunha CB. Consistência entre fontes de dados e confiabilidade interobservador do estudo da morbi-mortalidade e atenção peri e neonatal no município do Rio de Janeiro. Cad Saúde Publica. 2004; 20(Supl 1):S34-S43.

19. Ravid R. Practical statistics for educators. New York: University Press of America; 2000.

20. São Paulo (Município). Centro de Vigilância Sanitária. Portaria n²535, de 2003. Dispõe sobre o regulamento técnico para o controle higiênico-sanitário em empresas de alimentos, estabelecendo critérios e parâmetros para a produção de alimentos e bebidas, aplicados às empresas de alimentos. Diário Oficial do Município de São Paulo. 200324 out; Folha 16.

21. São Paulo (Estado). Centro de Vigilância Sanitária. Portaria n.6, de 10 de março de 1996. Dispõe sobre regulamento técnico, parâmetros e critérios para o controle higiênico-sanitário em estabelecimentos 
de alimentos. Diário Oficial do Estado de São Paulo. 199912 mar; Seção I, p.24.

22. Medeiros LC, Hillers VN, Chen G, Bergman V, Kendall $\mathrm{P}$, Schoeder M. Design and development of food safety and attitude scales for consumer food safety education. J Am Diet Assoc. 2004; 104(11):1671-7.

23. Farias Jr JC, Pires MC, Lopes AS. Reprodutibilidade de um questionário para o levantamento de informações sobre comportamentos relacionados à saúde em adolescentes. Rev Bras Cienc Mov. 2002; 10(3):43-8.

24. Scheule B. Food safety education: health professionals' knowledge and assessment of WIC client needs. J Am Diet Assoc. 2004; 104(5): 799-803.

25. Scheule B. Food-safety educational goals for dietetics and hospitality students. J Am Diet Assoc. 2000; 100(8):919-27.

26. Strohbehn CH, Gilmore SA, Sneed J. Food safety practices and HACCP implementation: perceptions of registered dietitians and dietary managers. J Am Diet Assoc. 2004; 104(11):1692-9.

27. Byrd-Bredbenner C. Food preparation knowledge and attitudes of young adults. Implications for nutrition practice. Top Clin Nutr. 2004; 19(2): 154-63.

28. Sneed J, Strohbehn C, Gilmore SA. Food safety practices and readiness to implement HACCP programs in assisted-living facilities in lowa. J Am Diet Assoc. 2004; 104(11):1678-83.

29. Haapala I, Probart C. Food safety knowledge, perceptions and behaviors among midle school students. J Nutr Educ Behav. 2004; 36(2):71-6.

30. Salvo VLMA, Gimeno SGA. Reprodutibilidade e validade do questionário de frequência de consumo de alimentos. Rev Saúde Publica. 2002; 36(4): 505-12.

Recebido em: 23/3/2006

Versão final reapresentada em: 10/11/2006 Aprovado em: 22/12/2006 